\title{
Molecular Detection and Identification of Babesia spp., Theileria spp., and Anaplasma spp. in Sheep From Border Regions, Northwestern China
}

\section{OPEN ACCESS}

Edited by:

David Modry,

University of Veterinary and

Pharmaceutical Sciences

Brno, Czechia

Reviewed by:

Georg Gerhard Duscher,

University of Veterinary Medicine,

Vienna, Austria

Zhisheng Dang,

National Institute of Parasitic

Diseases, China

*Correspondence: Xuenan Xuan

gen@obihiro.ac.jp

Bayin Chahan

2514062881@qq.com

Specialty section:

This article was submitted to

Parasitology

a section of the journal

Frontiers in Veterinary Science

Received: 02 March 2020

Accepted: 03 August 2020

Published: 16 September 2020

Citation:

Li Y, Galon EM, Guo Q, Rizk MA,

Moumouni PFA, LiU M, Li J, Ji S,

Chahan B and Xuan X (2020)

Molecular Detection and Identification

of Babesia spp., Theileria spp., and

Anaplasma spp. in Sheep From

Border Regions, Northwestern China.

Front. Vet. Sci. 7:630

doi: 10.3389/fvets.2020.00630

\author{
Yongchang Li ${ }^{1}$, Eloiza May Galon ${ }^{1}$, Qingyong Guo ${ }^{2}$, Mohamed Abdo Rizk ${ }^{1,3}$, \\ Paul Franck Adjou Moumouni ${ }^{1}$, Mingming Liu ${ }^{1}$, Jixu Li ${ }^{1}$, Shengwei Ji ${ }^{1}$, Bayin Chahan ${ }^{2 *}$ \\ and Xuenan Xuan ${ }^{1 *}$ \\ ${ }^{1}$ National Research Center for Protozoan Diseases, Obihiro University of Agriculture and Veterinary Medicine, Obihiro, Japan, \\ ${ }^{2}$ Parasitology Laboratory, Veterinary College, Xinjiang Agricultural University, Urumqi, China, ${ }^{3}$ Department of Internal \\ Medicine and Infectious Diseases, Faculty of Veterinary Medicine, Mansoura University, Mansoura, Egypt
}

Babesia, Theileria, and Anaplasma are important causative agents of tick-borne diseases that severely affect sheep. However, there is paucity in the occurrence genetic diversity of the infections of tick-borne diseases in sheep in border regions, northwestern China. In this study, nested polymerase chain reaction (nPCR) assays and gene sequencing were used to identify tick-borne Babesia spp., Theileria spp., and Anaplasma spp. infections in border regions, northwestern China. Out of 323 samples tested in this study, $225(69.7 \%)$ sheep were infected with Babesia spp., Theileria spp., and Anaplasma spp. Two hundred six (63.8\%), 60 (18.6\%), 54 (16.7\%), 51 (15.8\%), 32 (9.9\%), 19 (5.9\%), and 16 (5.0\%) were positive for A. ovis, B. motasi-like, A. bovis, T. uilenbergi, A. phagocytophilum, T. luwenshuni, and B. motasi-like Xinjiang, respectively. The most common dual infection was with $A$. ovis and $B$. motasi-like while the most frequent triple coinfection was $A$. ovis, B. motasi-like, and T. uilenbergi with coinfection rates of $17.0 \%$ (55/323) and 5.0\% (16/323), respectively. Sequencing analysis indicated that A. ovis MSP4, A. phagocytophilum epank1, A. bovis 16S rRNA, B. motasi-like rap1-b, B. motasi-like Xinjiang rap1-a, T. luwenshuni 18S rRNA, and T. uilenbergi 18S rRNA from border regions, northwestern China, showed 99-100\% identity with documented isolates from other countries. To the best of the authors' knowledge, this is the first report of T. uilenbergi and T. luwenshuni infections of sheep in border regions, northwestern China. Furthermore, these findings provide important data for understanding the distribution of Babesia, Theileria, and Anaplasma in sheep between border countries and China.

Keywords: Theileria, Babesia, Anaplasma, sheep, China 


\section{INTRODUCTION}

Babesiosis, theileriosis, and anaplasmosis are important diseases caused by pathogens transmitted by ticks (1). Two generally species of Babesia species (Babesia motasi and B. ovis) $(2,3)$ and several species of Theileria (Theileria ovis, T. lestoquardi, T. sespecies, T. luwenshuni, and T. uilenbergi) of ovine babesiosis and theileriosis are distributed in Asia, Africa, Europe, and the Far East (4-6). Furthermore, ovine anaplasmosis is mainly caused by $A$. ovis and $A$. marginale $(7,8)$. Clinical signs of babesiosis, theileriosis, and anaplasmosis in sheep and goats include anemia, fever, abortion, weight loss, reduce milk supply, jaundice, and even death, causing huge economic losses for sheep, and goat production $(3,5,7)$.

In China, Babesia motasi, B. ovis (9, 10), Theileria ovis, T. uilenbergi, T. luwenshuni (4-6), Anaplasma phagocytophilum, A. marginale, A. ovis, A. bovis, A. capra, and A. platys $(11,12)$ are the causative agents of ovine tick-borne disease transmitted by ticks or small ruminant that have been reported.

Xinjiang Uygur Autonomous Region (XUAR), an area surrounded by multiple land forms including Gobi desert, valley, mountain, grassland, and plates, occupies one-sixth of China's land area and borders eight countries including Russia, Mongolia, Kazakhstan, Kyrgyzstan, Tajikistan, Afghanistan, Pakistan, and India (13). According to Song et al. (14), in 11 border counties of XUAR, five Babesia species, two Theileria species and A. ovis were identified in Haemaphysalis punctata, Hyalomma asiaticum, Dermacentor nuttalli, D. marginatus, Rhipicephalus turanicus. Furthermore, in 2010, there were more than 30 million sheep and goats in XUAR (15), but information about ovine tick-borne pathogens is still lacking in border regions, northwest China. Subsequently, this study was conducted to help in full filling the information gap regarding the occurrence and genetic diversity of Babesia spp., Theileria spp., and Anaplasma spp. in sheep from northern and southern XUAR.

\section{MATERIALS AND METHODS}

Sheep blood samples $(n=323)$ were collected from Fuhai $(\mathrm{FH}$, $n=29$ ), Qinghe (QH, $n=81$ ), Jimunai (JM, $n=22)$, Bole (BL, $n=34$ ), Tashikurgan (TS, $n=76$ ), and Yecheng (YC, $n=81$ ) between June 2018 and June 2019 in the southern and northern XUAR (Figure 1). The age ranges of sampled sheep were 113 (0$\leq 2$ years old), 184 ( $>2-<7$ years old), and 26 ( $\geq 7$ years old) in 292 female and 31 male sheep. After collection, blood samples were transported to the laboratory in cool boxes and kept at $4^{\circ} \mathrm{C}$. DNA was extracted from $200 \mu \mathrm{l}$ of whole blood using the QIAamp DNA Blood Mini Kit (Qiagen, Germany), following the manufacturer's protocol and stored at $-30^{\circ} \mathrm{C}$ until used.

All samples were screened with species-specific primers for Theileria spp., Babesia spp., and Anaplasma spp. by PCR or nested PCR (nPCR) (Table S1). Based on fragments of major surface protein 4 (MSP4) gene of $A$. ovis and A. marginale, epank1 gene of $A$. phagocytophilum, 16S rRNA gene of $A$. bovis and A.capra, 18 ssu rRNA gene of $B$. ovis and $T$. ovis, rhoptry-associated protein (rap)1-b gene of $B$. motasilike, rap1-a gene of $B$. motasi-like Xinjiang, $18 \mathrm{~S}$ rRNA of
T. luwenshuni and T. uilenbergi, and major piroplasm surface protein (MPSP) of T. orientalis. The PCR reaction mixture was composed of the following Takara super mix (Takara, Japan), PCR products were checked by electrophoresis in 1.5\% agarose gels. Five positive samples were randomly selected from the six sampling areas, and at least three clones were sequenced. When the sequences obtained from different amplicons were identical, only one amplicon was retained for sequence analysis. Representative amplicons were cloned in pGEM-T Easy Vector. In brief, after extracting the amplicons from the agarose using QIAquick Gel Extraction Kit (Qiagen, Germany), pGEMT Easy Vector (Promega, USA), and NucleoSpin ${ }^{\circledR}$ Plasmid QuickPure (Macherey-Nagel, Germany). BigDye Terminator Cycle Sequencing Kit (Applied Biosystems, USA) and 3100 Genetic Analyzer (Applied Biosystems, USA) were used for sequencing (13). The nucleotide sequence identities were determined by performing GenBank BLASTn analysis on the NCBI database. The confidence interval (95\% CI) analyses were conducted using GraphPad Prismer 6.0.

\section{RESULTS AND DISCUSSION}

Among the 323 samples, the PCR assays revealed that 225 (69.7\%) sheep were infected with Babesia spp., Theileria spp., and Anaplasma spp. The overall infection rates of sheep are presented in Table 1. The most frequent pathogens were A. ovis detected in $63.8 \%$ of the samples, followed by B. motasi-like (18.6\%), A. bovis (16.7\%), T. uilenbergi (15.8\%), A. phagocytophilum (9.9\%), T. luwenshuni (5.9\%), and B. motasi-like Xinjiang (5.0\%) (Table 1). The most common coinfection was $A$. ovis and $B$. motasi-like with an infection rate of $17.0 \%$ (55/323), followed by $A$. ovis and T. uilenbergi with $12.4 \%$ (40/323). Meanwhile, only one sheep was dual infected with A. phagocytophilum and T. luwenshuni. For triple coinfection, the infection rate of $A$. ovis and B. motasi-like and T. uilenbergi was $5.0 \%$ (16/323) (Table S2).

All A. ovis, A. bovis, A. phagocytophilum, B. motasi-like, B. motasi-like Xinjiang, T. luwenshuni, and T. uilenbergi sequences in this study were the expected sizes of $347,551,444,536,507$, 389 , and $388 \mathrm{bp}$, respectively. The MSP4 gene sequences of $A$. ovis (GenBank accession numbers MN946542) were 97.9\% identical to other and to an isolate from Sudan (KU497712). A. bovis 16S rRNA gene sequences (GenBank Accession no. MN947620) was $99 \%$ identical to an isolate obtained from cattle in China (MK345480). A. phagocytophilum epank1 gene sequences (GenBank Accession no. MN946539) were 98\% identical to an isolate obtained from sheep in Germany (GU236795). Sequences of B. motasi-like rap1-b sequences (GenBank Accession nos. MN946540) showed 99\% identity to a previous isolate from XUAR sheep (KU510048). The sequence identity of B. motasilike Xinjiang rap1-a (GenBank Accession no. MN946541) with an isolate from Haemaphysalis longicornis in Gansu, China (KX708614), was 99\%. The accession numbers for 18S rRNA of T. luwenshuni and T. uilenbergi are MN944535 and MN944557, respectively. The complete list of GenBank accession numbers of sequences from this study is shown in Table S3. 


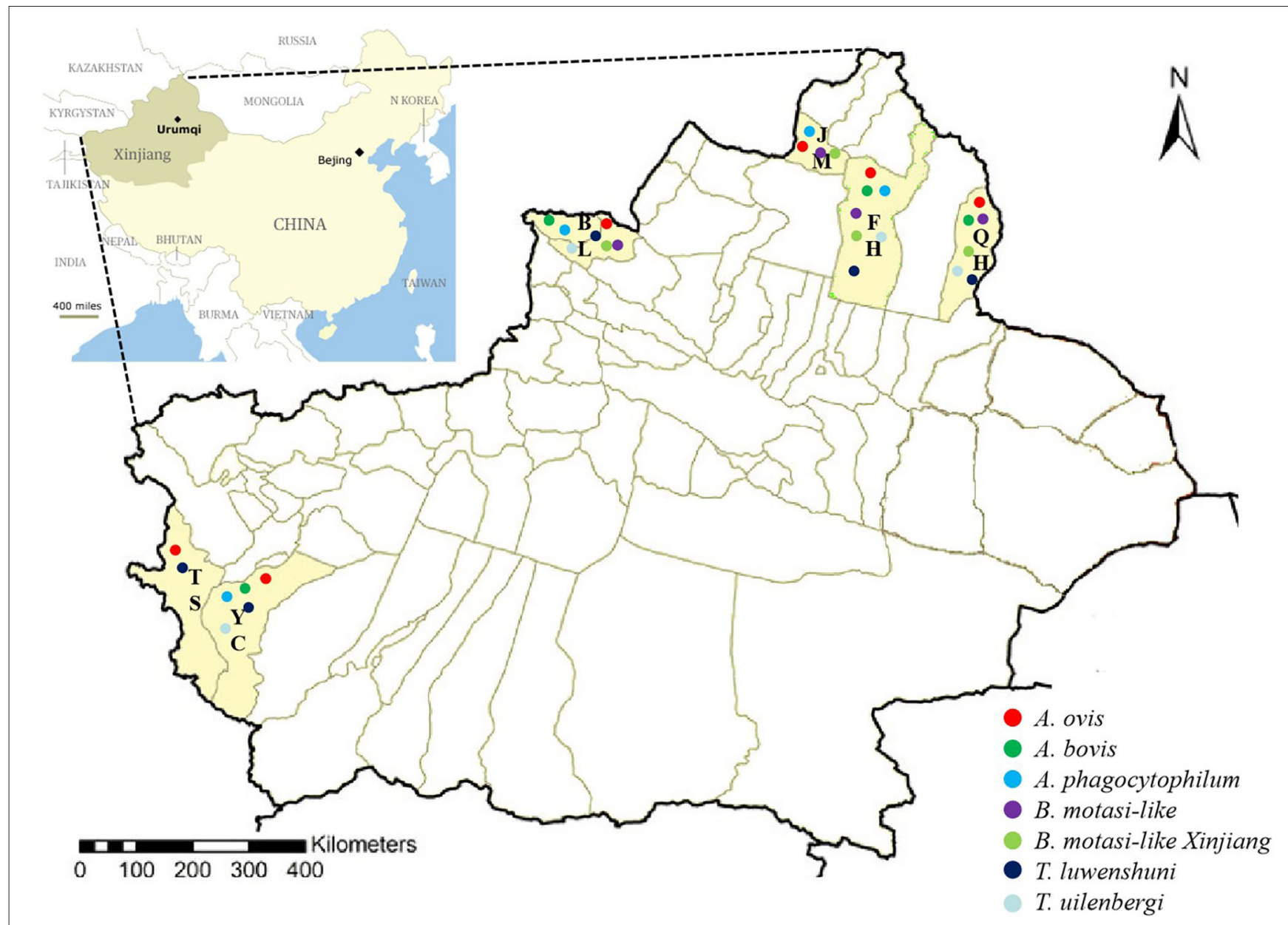

FH: Fuhai (29); QH: Qinghe (81); JM: Jimunai (22); BL: Bole (34); TS: Tashikurgan (76); YC: Yecheng (81)

FIGURE 1 | Map of XUAR, China. Dots indicate localities which pathogens were detected.

Tick-borne pathogens (TBPs) of ovines represent a serious threat to veterinary and public health worldwide (16-18). In this study, three species of Anaplasma and two species of Babesia and Theileria were molecularly detected from sheep. The result also revealed that $69.7 \%$ of the samples were infected with at least one pathogen. Generally, the relatively high incidence of TBPs could be attributed to the abundance of vectors in the study area. In XUAR, 45 species from 6 genera of ticks, namely, Hyalomma, Dermacentor, Haemaphysalis, and Rhipicephalus are reported (19). In addition, Rhipicephalus turanicus, Dermacentor niveus, Hyalomma asiaticum, and Dermacentor marginatus are the most frequent tick species in domestic animals from thirtyfive counties (cities) in XUAR during 2011-2017 (20).

A number of Anaplasma species including A. ovis, A. bovis, and $A$. phagocytophilum, have been documented to cause anaplasmosis in ruminants (21). In this study, we identified $A$. ovis (63.8\%), A. bovis (16.7\%), and A. phagocytophilum (9.9\%). Previously, (11) found A. ovis and A. phagocytophilum in sheep in XUAR, while A. ovis was identified in ticks in border regions
(14). In addition, $A$. ovis and $A$. bovis were identified in Fuhai and Qinghe while A. phagocytophilum was detected in Fuhai, which both border Mongolia. A. ovis was identified in sheep and Dermacentor nuttalli tick (22) and A. phagocytophilum in I. persulcatus in areas surrounding Mongolia (23). We also detected A. ovis, A. bovis, and A. phagocytophilum in Bole and $A$. ovis and $A$. phagocytophilum in Jimunai which both areas are adjoined to Kazakhstan. According to Shpynov et al. (24), A. phagocytophilum DNA was identified in I. persulcatus ticks in the Altai and Primorye territories, which is also borders Kazakhstan. Furthermore, A. ovis (79.0\%), A. bovis $(24.7 \%)$, and A. phagocytophilum (28.4\%) in Yecheng sheep in current study, while A. ovis (1.5\%), A. marginale (5.7\%), and A. centrale $(2.7 \%)$ were identified in ticks from Pakistan, the country that Yecheng borders (25). Other border countries of XUAR also have reports about Anaplasma infection (A. bovis, A. centrale, A. phagocytophilum, and $A$. marginale) in ruminants or ticks including Russia (26), Philippines (27), and south India (28). Notably, A. capra, a newly discovered emerging zoonotic species 
TABLE 1 | Detection of Anaplasma spp., Babesia spp., and Theileria spp. in sheep from XUAR, China.

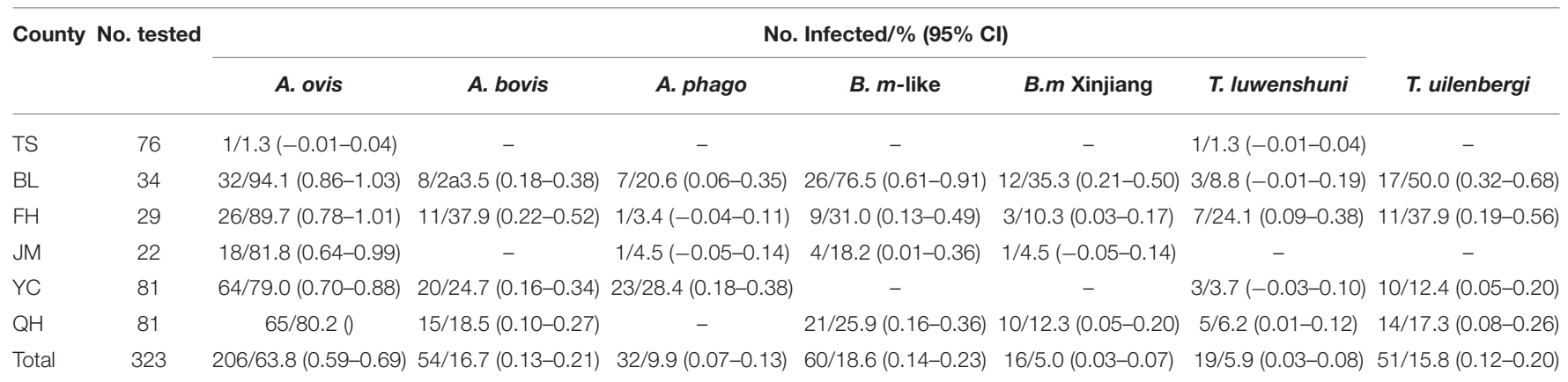

"-" mean not detected; TS, Tashikurgan; BI, Bole; FH, Fuhai; JM, Jimunai; YC, Yecheng; and QH, Qinghe.

of Anaplasma, was not identified in the current study, although it has been reported in both XUAR (only four positive samples) and Gansu of China $(29,30)$. Such discrepant is attributed to the difference in the area where the samples were collected in our study and in the previous one in XUAR (29).

Several Babesia species (B. motasi-like and B. ovis) are widely spread among sheep and goat in China, specifically in Gansu and Qinghai $(31,32)$, consistent with the distribution of the tick vectors (33). The total infection rates were $0.4 \%$ for $B$. motasi in sheep from Gansu according to (34). Song et al. (14) reported that in XUAR, five Babesia were identified in H. asiaticum, $H$. punctata, D. nuttalli, and $R$. turanicus ticks which were collected from sheep, including B. occultans, B. motasi-like, and $B$. major. Meanwhile, none of the samples was positive for $B$. motasi-like in ticks which were collected from Fuhai, Jimunai, Qinghe, and Yecheng (14). However, except in Yecheng, we found B. motasi-like infection in sheep in Fuhai (31.0\%), Jimunai (18.2), and Qinghe (25.9\%). According to Niu et al. (31), the recorded infection rate for $B$. motasi-like was $21.87 \%$, while Song et al. (14) also reveal that B. motasi-like was identified in $H$. punctata ticks in Yili district. In countries nearby XUAR, such as Pakistan, B. ovis (16\%) and T. ovis (23\%) were also detected from sheep (35).

The first infection by Theileria spp. in small ruminants imported into Sichuan province of China was reported in 1958 (36). Infection with other Theileria species, such as T. uilenbergi, T. luwenshuni, and T. ovis, infective to small ruminants, has been documented since the first report (34). Furthermore, T. luwenshuni and T. uilenbergi were found in Gansu and Sichuan, Heilongjiang, Qinghai, Hubei, and Hainan, China (12, 16, 32). In this study, pathogenic T. luwenshuni was detected in sheep from five counties (Fuhai, Bole, Qinghe, Yecheng, Tashikurgan), with detection rates of $24.1,8.8,6.2,3.7$, and $1.3 \%$, respectively. Although T. ovis (7.25\%) was previously detected in $R$. turanicus ticks infesting sheep in Yecheng (14), we only identified $T$. luwenshuni and T. uilenbergi in sheep from Yecheng in this study. T. lestoquardi was also found infecting apparently healthy sheep and goats from two districts of Pakistan, which was near Yecheng county (37). In addition, T. luwenshuni infection in ticks from dogs and cattle was identified by reverse line blot hybridization (34). Aktaş et al.
(38) revealed the presence of T. orientalis and T. annulata from cattle in Kyrgyzstan, which is near Bole. In south India, which is near Yecheng and Tashikurgan, researchers also identified T. orientalis in ticks (29). In previous studies, T. ovis were found in ticks (39) and goats (40) from XUAR, but in the present study, all sheep DNA samples showed a negative result for T. ovis and T. orientalis. The reasonable explanation for such obtained finding might be attributed to the difference between collection sample areas and tick species with previous study in XUAR.

The distribution and diversity of pathogens in sheep may be attributed to the difference in macroclimate, tick species, tickdwelling habitat, and landscape between districts. Particularly, questing ticks can be found in habitats like herb layer and vegetation $(41,42)$. In XUAR, cattle, horses, and sheep often co-feed in the same district, and there are also many small mammals in areas with high vegetation (43). These expand the possibility of ticks finding an animal as its host, but reduce the possibility of ticks encountering the same animal (44). Furthermore, the districts containing alpine and meadow climates have humid soils rich in organic matter resulting in abundant vegetation which favors the tick population $(42,45)$. Those aspects might explain why a lot of TBPs and different tick species were identified in XUAR. Although this study screened several TBPs in sheep reared in the southern and northern parts of XUAR, China, this survey did not include ticks from this area. Therefore, other future studies are required to detect those pathogens in tick spp. collected from different parts of XUAR. Furthermore, the present study did not determine the risk factors associated with screened pathogens on either animal or farm level. Subsequently, more studies are warranted in the future to avoid the risk factors correlated with TBPs in XUAR.

This study revealed the presence of $A$. ovis, A. bovis, A. phagocytophilum B. motasi-like, B. motasi-like Xinjiang, T. uilenbergi, and T. luwenshuni in sheep from six border counties (Qinghe, Fuhai, Jimunai, Bole, Tashikuergan, Yecheng) of XUAR, China. The current results present valuable information about ovine tick-borne infections in XUAR, which might suggest the possible emergence of tick-borne diseases in small ruminants in border regions of northwest China. 
Moreover, information generated from this study is expected to improve the approach for prevention and control of tick-borne diseases in China.

\section{DATA AVAILABILITY STATEMENT}

Publicly available datasets were analyzed in this study. This data can be found in the NCBI: MN946542, MN947620, MN946539, MN946540, MN946541, MN944535, and MN944557.

\section{ETHICS STATEMENT}

The animal study was reviewed and approved by the owners of the selected farms were informed of the study and provided their written approval for sampling of their sheep. All procedures were carried out according to the ethical guidelines for the use of animal samples permitted by Obihiro University of Agriculture and Veterinary Medicine (Approval ID: 18-40). Written informed consent was obtained from the owners for the participation of their animals in this study.

\section{AUTHOR CONTRIBUTIONS}

YL: methodology, validation, statistical analysis of the results, writing-original draft, and writing-review \& editing.

\section{REFERENCES}

1. Zhou M, Cao S, Sevinc F, Sevinc M, Ceylan O, Moumouni PFA, et al. Molecular detection and genetic identification of Babesia bigemina, Theileria annulata, Theileria orientalis and Anaplasma marginale in Turkey. Ticks Tick Borne Dis. (2015) 1:126-34. doi: 10.1016/j.ttbdis.2015.09.008

2. Schnittger L, Rodriguez AE, Florin-Christensen M, Morrison DA. Babesia: a world emerging. Infect Genet Evol. (2012) 8:1788-809. doi: 10.1016/j.meegid.2012.07.004

3. Yin H, Lu W, Luo J. Babesiosis in China. Trop Anim Health Prod. (1997) 4:11S-5S. doi: 10.1007/BF02632908

4. Gebrekidan H, Hailu A, Kassahun A, Rohoušová I, Maia C, Talmi-Frank D, et al. Theileria infection in domestic ruminants in northern Ethiopia. Vet. Parasitol. (2014) 200:31-8. doi: 10.1016/j.vetpar.2013.11.017

5. Berggoetz M, Schmid M, Ston D, Wyss V, Chevillon C, Pretorius AM, et al. Tick-borne pathogens in the blood of wild and domestic ungulates in South Africa: interplay of game and livestock. Ticks Tick Borne Dis. (2014) 2:166-75. doi: 10.1016/j.ttbdis.2013.10.007

6. Zhou M, Cao S, Sevinc F, Sevinc M, Ceylan O, Ekici S, et al. Molecular detection and genetic characterization of Babesia, Theileria and Anaplasma amongst apparently healthy sheep and goats in the central region of Turkey. Ticks Tick Borne Dis. (2017) 2:246-52. doi: 10.1016/j.ttbdis.2016.11.006

7. Splitter EJ, Twiehaus MJ, Castro ER. Anaplasmosis in sheep in the United States. J Am Vet Med Assoc. (1955) 942:244-5.

8. Ciani E, Alloggio I, Petazzi F, Pieragostini E. Looking for prognosticators in ovine anaplasmosis: discriminant analysis of clinical and haematological parameters in lambs belonging to differently susceptible breeds experimentally infected with Anaplasma ovis. Acta Vet Scand. (2013) 1:71. doi: 10.1186/1751-0147-55-71

9. Wang H, Yang J, Mukhtar MU, Liu Z, Zhang M, Wang X. Molecular detection and identification of tick-borne bacteria and protozoans in goats and wild Siberian roe deer (Capreolus pygargus) from Heilongjiang Province, northeastern China. Parasit Vectors. (2019) 1:296. doi: 10.1186/s13071-019-3553-1
EG: validation, statistical analysis the results, and writingreview \& editing. QG, MR, and PM: investigation and writing-review \& editing. ML, JL, and SJ: Investigation and recorded samples' data. BC: investigation, recorded samples' data, and funding acquisition. XX: conceptualization, writing-review \& editing, and funding acquisition. All authors contributed to the article and approved the submitted version.

\section{FUNDING}

This study was supported by a grant from Japan Society for the Promotion of Science (JSPS) Core to Core Program and National Natural Science Foundation of China (31660711).

\section{ACKNOWLEDGMENTS}

We are grateful to the farmers for their cooperation and the state veterinarians for helping in the blood sample collection in the field.

\section{SUPPLEMENTARY MATERIAL}

The Supplementary Material for this article can be found online at: https://www.frontiersin.org/articles/10.3389/fvets. 2020.00630/full\#supplementary-material

10. Li Y, Luo J, Guan G, Ma M, Liu A, Liu J, et al. Experimental transmission of Theileria uilenbergi infective for small ruminants by Haemaphysalis longicornis and Haemaphysalis qinghaiensis. Parasitol Res. (2009) 5:122731. doi: 10.1007/s00436-009-1347-7

11. Yang J, Li Y, Liu Z, Liu J, Niu Q, Ren Q, et al. Molecular detection and characterization of Anaplasma spp. in sheep and cattle from Xinjiang, Northwest China. Parasit Vectors. (2015) 8:108. doi: 10.1186/s13071-015-0727-3

12. Zhou Z, Li K, Sun Y, Shi J, Li H, Chen Y, et al. Molecular epidemiology and risk factors of Anaplasma spp., Babesia spp. and Theileria spp. infection in cattle in Chongqing, China. PLoS ONE. (2019) 7:e0215585. doi: 10.1371/journal.pone.0215585

13. Li Y, Li J, Chahan B, Guo Q, Zhang Y, Moumouni PFA, et al. Molecular investigation of tick-borne infections in cattle from Xinjiang Uygur autonomous Region, China. Parasitol Int. (2020) 74:101925. doi: 10.1016/j.parint.2019.05.003

14. Song R, Wang Q, Guo F, Liu X, Song S, Chen C, et al. Detection of Babesia spp., Theileria spp. and Anaplasma ovis in border regions, Northwestern China. Transbound Emerg Dis. (2018) 6:1537-44. doi: 10.1111/tbed.12894

15. Statistic bureau of Xinjiang Uygur Autonomous Region. (2011). Available online at: http://www.xjtj.gov.cn/tjfx/201404/t20140419_429995.html (accessed September 19, 2019).

16. Yin H, Schnittger L, Luo J, Seitzer U, Ahmed JS. Ovine theileriosis in China: a new look at an old story. Parasitol Res. (2007) 101:1915. doi: 10.1007/s00436-007-0689-2

17. Ben MS, Belkahia H, Messadi L. Anaplasma spp. in North Africa: A review on molecular epidemiology, associated risk factors and genetic characteristics. Ticks Tick Borne Dis. (2018) 3:543-55. doi: 10.1016/j.ttbdis.2018. 01.003

18. Sadeddine R, Diarra AZ, Laroche M, Mediannikov O, Righi S, Benakhla A, et al. Molecular identification of protozoal and bacterial organisms in domestic animals and their infesting ticks from north-eastern Algeria. Ticks Tick Borne Dis. (2019) 2:101330. doi: 10.1016/j.ttbdis.2019.1 01330 
19. Zhang Y, Chahan B, Liu S, Song R, Li Y, Huercha, et al. Epidemiologic studies on Theileria equi infections for grazing horses in Ili of Xinjiang province. Vet Parasitol. (2017) 244:111-113. doi: 10.1016/j.vetpar.2017.07.014

20. Sheng J, Jiang M, Yang M, Bo X, Zhao S, Zhang Y, et al. Tick distribution in border regions of Northwestern China. Ticks Tick Borne Dis. (2019) 3:6659. doi: 10.1016/j.ttbdis.2019.02.011

21. Rar V, Golovljova I. Anaplasma, Ehrlichia, and "Candidatus Neoehrlichia" bacteria: pathogenicity, biodiversity, and molecular genetic characteristics, a review. Infect Genet Evol. (2011) 8:1842-61. doi: 10.1016/j.meegid.2011.09.019

22. Enkhtaivan B, Narantsatsral S, Davaasuren B, Otgonsuren D, Amgalanbaatar T, Uuganbayar E, et al. Molecular detection of Anaplasma ovis in small ruminants and ixodid ticks from Mongolia. Parasitol Int. (2019) 69:4753. doi: 10.1016/j.parint.2018.11.004

23. Jiang J, Jiang B, Yu J, Zhang W, Gao H, Zhan L, et al. Anaplasma phagocytophilum infection in ticks, China-Russia border. Emerging Infect Dis. (2011) 5:932-4. doi: 10.3201/eid1705.101630

24. Shpynov SN, Rudakov NV, Iastrebov VK, Leonova GN, Khazova TG, Egorova NV, et al. New evidence for the detection of Ehrlichia and Anaplasma in ixodes ticks in Russia and Kazakhstan. Med Parazitol. (2004) 2:10-4.

25. Rehman A, Conraths FJ, Sauter-Louis C, Krücken J, Nijhof AM. Epidemiology of tick-borne pathogens in the semi-arid and the arid agro-ecological zones of Punjab province, Pakistan. Transbound Emerg. (2019) 1:52636. doi: 10.1111/tbed.13059

26. Livanova NN, Fomenko NV, Akimov IA, Ivanov MJ, Tikunova NV, Armstrong R, et al. Dog survey in Russian veterinary hospitals: tick identification and molecular detection of tick-borne pathogens. Parasit Vectors. (2018) 1:591. doi: 10.1186/s13071-018-3161-5

27. Galon EMS, Moumouni PFA, Ybañez RHD, Ringo AE, Efstratiou A, Lee SH, et al. First molecular detection and characterization of tick-borne pathogens in water buffaloes in Bohol, Philippines. Ticks Tick Borne Dis. (2019) 4:81521. doi: 10.1016/j.ttbdis.2019.03.016

28. Nimisha M, Devassy JK, Pradeep RK, Pakideery V, Sruthi MK, Pious A, et al. Ticks and accompanying pathogens of domestic and wild animals of Kerala, South India. Exp Appl Acarol. (2019) 1:137-55. doi: 10.1007/s10493-019-00414-Z

29. Peng Y, Wang K, Zhao S, Yan Y, Wang H, Jing J, et al. Detection and phylogenetic characterization of Anaplasma capra: an emerging pathogen in sheep and goats in China. Front Cell Infect Microbiol. (2018) 8:283. doi: 10.3389/fcimb.2018.00283

30. Yang J, Han R, Niu Q, Liu Z, Guan G, Liu G, et al. Occurrence of four Anaplasma species with veterinary and public health significance in sheep, northwestern China. Ticks Tick Borne Dis. (2018) 1:825. doi: 10.1016/j.ttbdis.2017.10.005

31. Niu Q, Valentin C, Bonsergent C, Malandrin L. Strong conservation of rhoptryassociated-protein-1 (RAP-1) locus organization and sequence among Babesia isolates infecting sheep from China (Babesia motasi-like phylogenetic group). Infect Genet Evol. (2014) 28:21-32. doi: 10.1016/j.meegid.2014.08.028

32. Li J, Ma L, Moumouni PFA, Jian Y, Wang G, Zhang X, et al. Molecular survey and characterization of tick-borne pathogens in sheep from Qinghai, China. Small Rumin Res. (2019) 175:23-30. doi: 10.1016/j.smallrumres.2019.01.014

33. Wang J, Ma M, Liu A, Ren Q, Li A, Liu Z, et al. A sero-epidemiological survey of Chinese Babesia motasi for small ruminants in China. Parasitol Res. (2013) 6:2387-91. doi: 10.1007/s00436-013-3310-x
34. Sun M, Wang J, Liu Z, Guan G, Li Y, Liu J, et al. First molecular evidence of Babesia occultans and Theileria separata infection in ticks and sheep in China. Exp Appl Acarol. (2019) 2:223-9. doi: 10.1007/s10493-01900369-1

35. Shahzad W, Noor H, Ahmad MU, Munir R, Sharif SM, Hassan MM, et al. Prevalence and molecular diagnosis of Babesia ovis and Theileria ovis in Lohi sheep at livestock experiment station (LES), Bahadurnagar, Okara, Pakistan. Iran. J Parasitol. (2013) 4:570-8.

36. Yang F, Feng Z, Yu G, Liu J, Wei Z, He X. A report on ovie theileriosis in ganning animal farm station in Ganzi District. Chin Vet Sci. (1958) 2:33-7.

37. Saeed S, Jahangir M, Fatima M, Shaikh RS, Khattak RM, Ali M, et al. PCR based detection of Theileria lestoquardi in apparently healthy sheep and goats from two districts in Khyber Pukhtoon Khwa (Pakistan). Trop Biomed. (2015) 2:225-32.

38. Aktaş M, Kisadere I, Özübek S, Cihan H, Salikov R, Cirak VY. First molecular survey of piroplasm species in cattle from Kyrgyzstan. Parasitol Res. (2019) 8:2431-5. doi: 10.1007/s00436-019-06370-2

39. Li Y, Guan G, Ma M, Liu J, Ren Q, Luo J, et al. Theileria ovis discovered in China. Exp Parasitol. (2011) 1:304-7. doi: 10.1016/j.exppara.2010.07.002

40. Yang Y, Mao Y, Kelly P, Yang Z, Luan L, Zhang J, et al. A panTheileria FRET-qPCR survey for Theileria spp. in ruminants from nine provinces of China. Parasit Vectors. (2014) 7:413. doi: 10.1186/1756-330 5-7-413

41. Randolph S, Storey K. Impact of microclimate on immature tickrodent host interactions (Acari: Ixodidae): implications for parasite transmission. J Med Entomol. (1999) 36:741-8. doi: 10.1093/jmedent/3 6.6.741

42. Ehrmann S, Ruyts SC, Lorenzen MS, Bauhus J, Brunet J, Ocusins SA, et al. Habitat properties are key drivers of Borrelia burgdorferi (s.l.) prevalence in Ixodes ricinus populations of deciduous forest fragments. Parasit Vectors. (2018) 1:23. doi: 10.1186/s13071-017-2590-x

43. Kollmann J, Buschor M. Edges effects on seed predation by rodents in deciduous forests of northern Switzerland. Plant Ecol. (2002) 164:24961. doi: 10.1023/A:1021225728493

44. Handa I, Aerts R, Berendse F, Berg M, Bruder A, Butenschoen O, et al. Consequences of biodiversity loss for litter decomposition across biomes. Nature. (2014) 7499:218-21. doi: 10.1038/nature13247

45. Schwarz A, Maier W, Kistemann T, Kampen H. Analysis of the distribution of the tick Ixodes ricinus. (Acari: Ixodidae) in a nature reserve of western Germany using geographic information systems. Int J Hyg Environ Health. (2009) 1:87-96. doi: 10.1016/j.ijheh.2007.12.001

Conflict of Interest: The authors declare that the research was conducted in the absence of any commercial or financial relationships that could be construed as a potential conflict of interest.

Copyright (c) 2020 Li, Galon, Guo, Rizk, Moumouni, Liu, Li, Ji, Chahan and Xuan. This is an open-access article distributed under the terms of the Creative Commons Attribution License (CC BY). The use, distribution or reproduction in other forums is permitted, provided the original author(s) and the copyright owner(s) are credited and that the original publication in this journal is cited, in accordance with accepted academic practice. No use, distribution or reproduction is permitted which does not comply with these terms. 